\title{
Dimensional Accuracy of 3D-printed Models of the Right First Metacarpal Bones of Cadavers
}

\author{
Ian Oliver D. Macatangay, ${ }^{1}$ Jessa Joy C. Malipot, ${ }^{1}$ Alyanna Marie M. Lopez, ${ }^{1}$ Robert Earl C. Mabulay, ${ }^{1}$ \\ Rodee Ann Kate O. Magpantay, ${ }^{1}$ Larysa S. Malecdan, ${ }^{1}$ Jana Louria M. Malingan, ${ }^{1}$ Grace Zurielle C. Malolos, ${ }^{1}$ \\ Philippe Angelo A. Mamaril, ${ }^{1}$ Angela Nicole M. Mananghaya ${ }^{1}$ and Rafael C. Bundoc ${ }^{2}$ \\ ${ }^{1}$ College of Medicine, University of the Philippines Manila \\ ${ }^{2}$ Department of Anatomy, College of Medicine, University of the Philippines Manila
}

\begin{abstract}
Background. The use of 3D printing in medical education, prosthetics, and preoperative planning requires dimensional accuracy of the models compared to the replicated tissues or organs.

Objective. To determine the dimensional accuracy of 3D-printed models replicated from metacarpal bones from cadavers.

Methods. Fifty-two models were 3D-printed using fused deposition modeling (FDM), stereolithography (SLA), digital light processing (DLP), and binder jetting method from 13 right first metacarpal bones of cadavers from the College of Medicine, University of the Philippines Manila. Six dimensional parameters of the 3D-printed models and their control bones were measured using $0.01 \mathrm{~mm}$ calipers - length, midshaft diameter, base width, base height, head width, and head height. Mean measurements were compared using non-inferiority testing and multidimensional scaling.

Results. Mean measurements of the 3D-printed models were slightly larger than their control bones (standard deviation range: 1.219-4.264; standard error range, 0.338-1.183). All models were found to be at least $90 \%$ accurate and statistically non-inferior compared to control bones. DLP-printed models were the most accurate (base width, $99.62 \%)$ and most similar to their control bone $(-0.05,90 \% \mathrm{Cl}-0.34,0.24)$. Through multidimensional scaling, DLP-printed models (coordinate $=0.437$ ) were the most similar to the control bone (coordinate $=0.899$ ).
\end{abstract}

Conclusion. The 3D-printed models are dimensionally accurate when compared to bones.

Key Words: Printing, 3D, three-dimensional, stereolithography, dimensional measurement, accuracy, templating, surgical planning

\section{INTRODUCTION}

Three-dimensional (3D) printing is an emerging tool in medicine that is mainly utilized for the personalization and customization of medical procedures. The current medical applications of $3 \mathrm{D}$ printing are vast and far-reaching from anatomical models and prosthetics, to biomedical research, medical education, and surgery. Current medical

Paper presented at the UP Manila Science and Technology Week: Machines and Tests Research Forum, August 29, 2019, College of Nursing Auditorium, University of the Philippines Manila.

Corresponding author: Ian Oliver D. Macatangay College of Medicine

University of the Philippines Manila

547 Pedro Gil St., Ermita, Manila 1000, Philippines

Email: idmacatangay@up.edu.ph applications of $3 \mathrm{D}$ printing are tissue and organ fabrication, anatomical models for prosthetic or pharmaceutical use, and customized or personalized medicine. ${ }^{1}$

In surgery, 3D printing is used preoperatively as a model for surgical templating of body parts or organs involved in the operation. This could lessen planning time and anatomical variation errors since the $3 \mathrm{D}$ model will serve as the main guide for the patient procedure. The use of $3 \mathrm{D}$ 
models by surgeons allows accurate preoperative planning, precise implant placement, and better surgical results. ${ }^{2}$

The digital acquisition of detailed 3D anatomic images is possible by the use of CT (computed tomography) scanning and computer-aided image-editing software. ${ }^{3}$ These renderings may be sized, oriented, and exported to manufacture replicas that may be used to facilitate the assessment and planning of complex medical and surgical cases.

Anatomical models are the cornerstone of medical education and are currently being utilized in surgery for accurate patient-specific surgical templating. Application of $3 \mathrm{D}$ printing technology in the diagnosis and treatment procedure of complex medical and surgical cases is helpful in multiple ways. ${ }^{4}$ It could make the diagnosis more accurate and the treatment more specific to the patient. Furthermore, 3D-printed models could facilitate communication between the physician and patients due to its visual characteristics. It also helps surgeons do preoperative design - reducing the duration of operation and amount of bleeding will improve the post-operative rehabilitation process significantly. Overall, 3D printed models help doctors observe, treat and communicate with patients more effectively.

Determination of accuracy of $3 \mathrm{D}$ models is essential to the success of a surgical operation or any medical procedure that utilizes these models for personalized treatment planning. It also provides quality assurance to the authenticity of the $3 \mathrm{D}$ model as a guide for surgeries and other medical applications such as prosthetics and anatomical models.

Metacarpal bones were chosen as control in this study because of the following reasons: existence of a standardized detailed instructions for measurement, relatively easy accessibility compared to other bones, preservation of the cadaveric skeleton for further academic purposes, and it is a small yet important bone since it helps in thumb opposition which is one of the fine motor skills of an individual.

With this study, the authors aimed to determine the accuracy of 3D-printed models of the right first metacarpal bones of cadavers from the UP College of Medicine.

\section{MATERIALS AND METHODS}

\section{Study Design and Setting}

To determine the accuracy of the 3D-printed models, an analytic study design was conducted by comparing the 3D-printed models to the cadaveric first metacarpal bones using six measurement parameters.

The study sample of right metacarpals was obtained from cadavers used by students for anatomic dissection at the Department of Anatomy, University of the Philippines College of Medicine (UPCM). Measurement of the bones was conducted at the Multidisciplinary Laboratory (MDL) rooms, Paz Mendoza Building, UP Manila. The bones and models underwent CT scan at the Radiology Center of the UP-Philippine General Hospital (PGH) Faculty Medical Arts Building (FMAB). The models were printed using four different 3D printers and 3D printing techniques at Mapua University.

\section{Population and Sampling Technique}

Out of the population of 30 cadavers, simple randomization sampling method was used to select 13 cadavers. The right first metacarpals of the selected cadavers were harvested and cleaned within a period of one week.

3D-printed models using four different printing methods were produced from each harvested metacarpal bone (control group), and served as the experimental group.

\section{Study Variables}

The dependent variable was dimensional accuracy, defined as the non-inferiority of the mean difference in measured parameters between the 3D-printed models and cadaveric bones. The independent variables included the type of $3 \mathrm{D}$ printer used and the dimensions which were based from the Scheuer and Elkington Measurements, were length, midshaft diameter, base width, base height, head width, and head height (Figure 1; Table 1)..$^{5}$

\section{Study procedure}

\section{Preparation of the 3D-printed models}

\section{Computed Tomography (CT) scan imaging}

Imaging of the 13 right first metacarpal bones was done using a CT scan (Siemens SOMATOM Definition AS and Syngo Acquisition Workplace) with $1 \mathrm{~mm}$ slice thickness, known to be the easiest modality for creating a model for $3 \mathrm{D}$ printing. The volumetric data obtained was then rendered in Digital Imaging and Communication in Medicine (DICOM) format.

\section{Conversion of DICOM image to STL format}

The DICOM image was subjected to segmentation, which involved extracting and isolating a region of interest

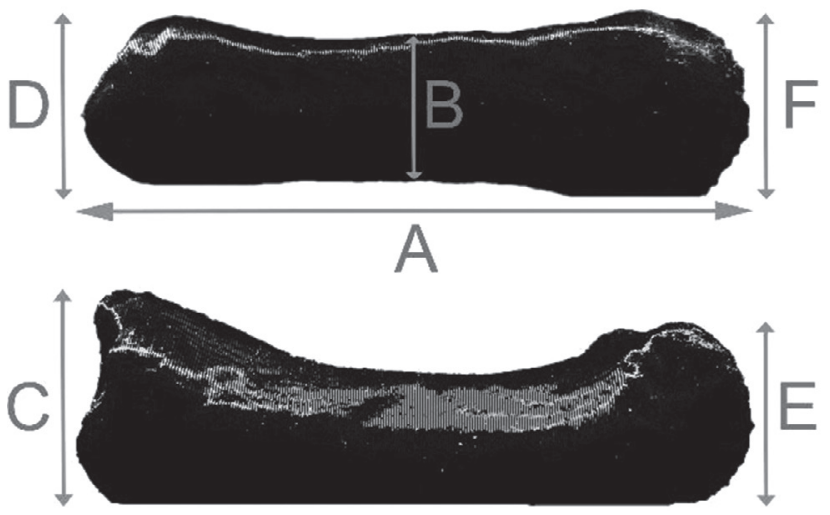

Figure 1. Dimensional parameters. Length (A), midshaft diameter (B), base height (C), base width (D), head height (E), and head width (F). 
Table 1. Measurement techniques for dimensional parameters

\begin{tabular}{|c|c|}
\hline Parameter & Measurement Technique \\
\hline Length & $\begin{array}{l}\text { Metacarpals were placed on their palmar surface, their long axis parallel to the long axis of the caliper. The proximal end rested } \\
\text { against the stationary upright. The dorsal surface of the distal end was kept relatively horizontal by holding it firmly near the base. }\end{array}$ \\
\hline $\begin{array}{l}\text { Midshaft } \\
\text { diameter }\end{array}$ & $\begin{array}{l}\text { This was done on the marked midpoint of the bone, i.e., one-half of the maximum axial length. With the bone resting its dorsal } \\
\text { surface on the table, its head directed toward the measurer and its base directed away, the mark was used at the midshaft to } \\
\text { guide the placement of the upper jaw of the calipers assuring that the calipers were held perpendicular to the long axis of the } \\
\text { bone and parallel with the table. The jaws of the caliper were closed until the lower jaw contacted the bone. }\end{array}$ \\
\hline Base height & $\begin{array}{l}\text { The metacarpal was held by its distal end such that the dorsal surface of the metacarpal base contacts the fixed upright, and } \\
\text { the long axis of the bone up to its midpoint is perpendicular to the long axis of the caliper. The measurement was taken with } \\
\text { approximately half of the shaft projecting from the far end of the caliper. }\end{array}$ \\
\hline Base height & $\begin{array}{l}\text { The metacarpal was held by its distal end such that the dorsal surface of the metacarpal base contacts the fixed upright, and } \\
\text { the long axis of the bone up to its midpoint is perpendicular to the long axis of the caliper. The measurement was taken with } \\
\text { approximately half of the shaft projecting from the far end of the caliper. }\end{array}$ \\
\hline Head width & $\begin{array}{l}\text { The metacarpal was held by its distal end such that the dorsal surface of the metacarpal base contacts the fixed upright, and } \\
\text { the long axis of the bone up to its midpoint is perpendicular to the long axis of the caliper. The measurement was taken with } \\
\text { approximately half of the shaft projecting from the far end of the caliper. }\end{array}$ \\
\hline Head height & $\begin{array}{l}\text { The metacarpal was held by holding it at its base with the dorsal surface of the head flush against the fixed upright, and the long } \\
\text { axis of the bone perpendicular to the long axis of the caliper. The dorsal part of the head usually contacted the stationary upright } \\
\text { at two points near the medial and lateral sides. The measurement was taken with approximately half of the shaft projecting from } \\
\text { the far end of the caliper. }\end{array}$ \\
\hline
\end{tabular}

(ROI) from the DICOM image. Mesh generation was also done, which served to extract a surface from the segmented data. These were achieved using the 3D Slicer software.

Images in DICOM format were then converted to Standard Tessellation Language or Stereolithography (STL), a format amenable for $3 \mathrm{D}$ printing, as the former could not be used for such purposes (Figure 2).

\section{STL post-processing}

After conversion to STL, post-processing and corrections on the mesh model were done to reduce errors that could affect the $3 \mathrm{D}$ printing accuracy. Interactive triangle mesh processing such as hole filling, mesh editing, smoothing,

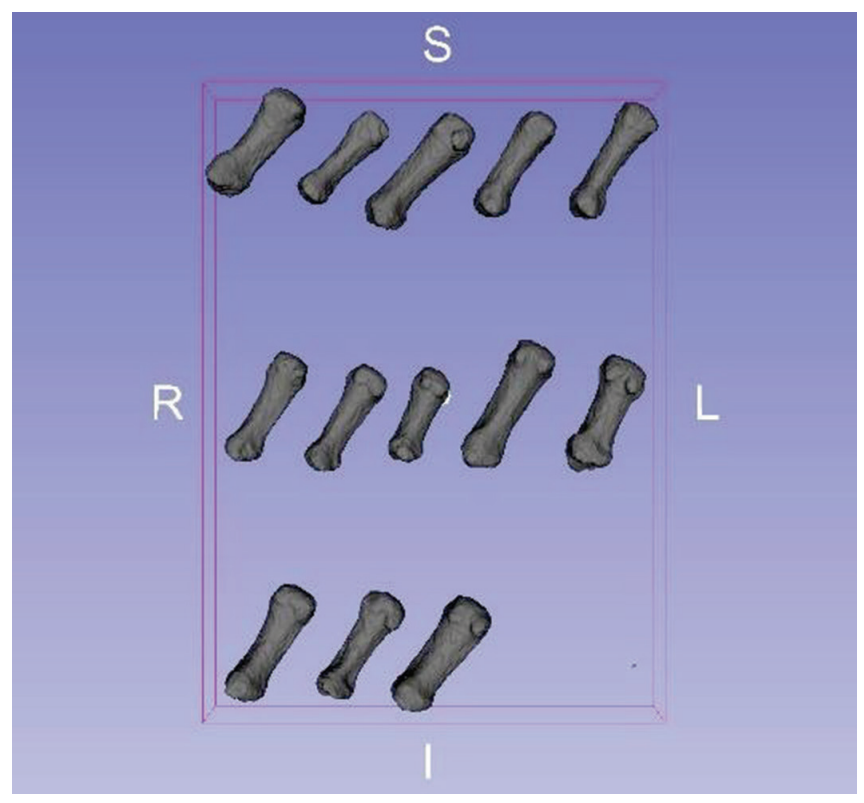

Figure 2. Stereolithography format of the bones. and self- intersecting inspection in the 3D Slicer software provided the means for any corrective measures that needed to be done.

\section{3D Printing}

The STL file was then used for 3D printing using four different methods as shown in Figure 3: standard fused deposition modeling (FDM), stereolithography (SLA), digital light processing (DLP), and binder jetting.

Acrylonitrile butadiene styrene (ABS) was the thermoplastic utilized in FDM due to its high durability and fine feature detail. A support structure (Figure 4) was also created to hold the 3D-printed model into its proper mold. Stereolithography and digital light processing, on the other hand, both utilized photoreactive liquid resin and support structures. Binder jetting, on the other hand, did not require a support structure and utilized white powder fused by binder material. Details of the different $3 \mathrm{D}$ printers are in Appendix. All the processes involved layer by layer printing until the $3 \mathrm{D}$-printed models were complete. All rafts were removed prior to measurements.

Volunteers who underwent skills training in measurement served as outcome assessors. The bones and the $3 \mathrm{D}$-printed models were randomly assigned to them. Only calipers that could measure up to $0.01 \mathrm{~mm}$ were used for each measurement. Each of the six parameters were measured in three replications for both cadaver-harvested metacarpal bones and $3 \mathrm{D}$-printed models. For each replication, there was a different outcome assessor. However, contrary to the techniques of Scheuer and Elkington that used miniosteometric board (MOB), ${ }^{5}$ this study used calipers since these were commercially available. The calipers used were all of the same brand, type, and size. All measurements were recorded to the nearest $0.01 \mathrm{~mm}$ and were recorded in Microsoft Excel 2016. 

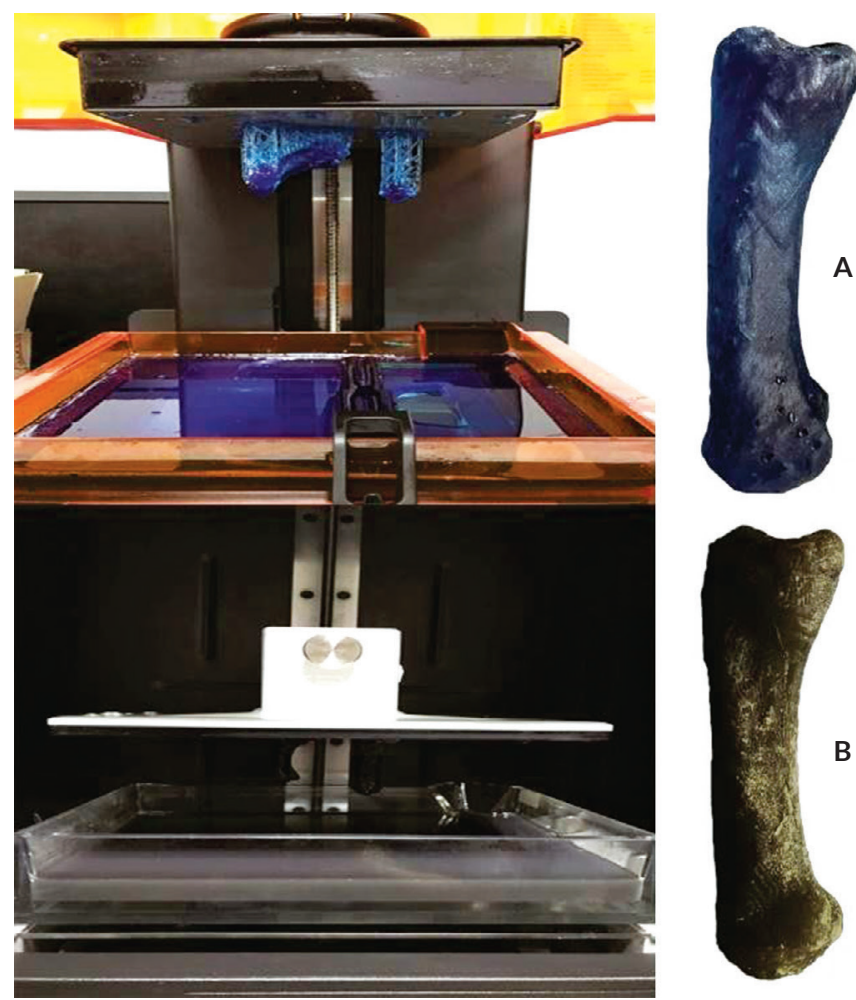
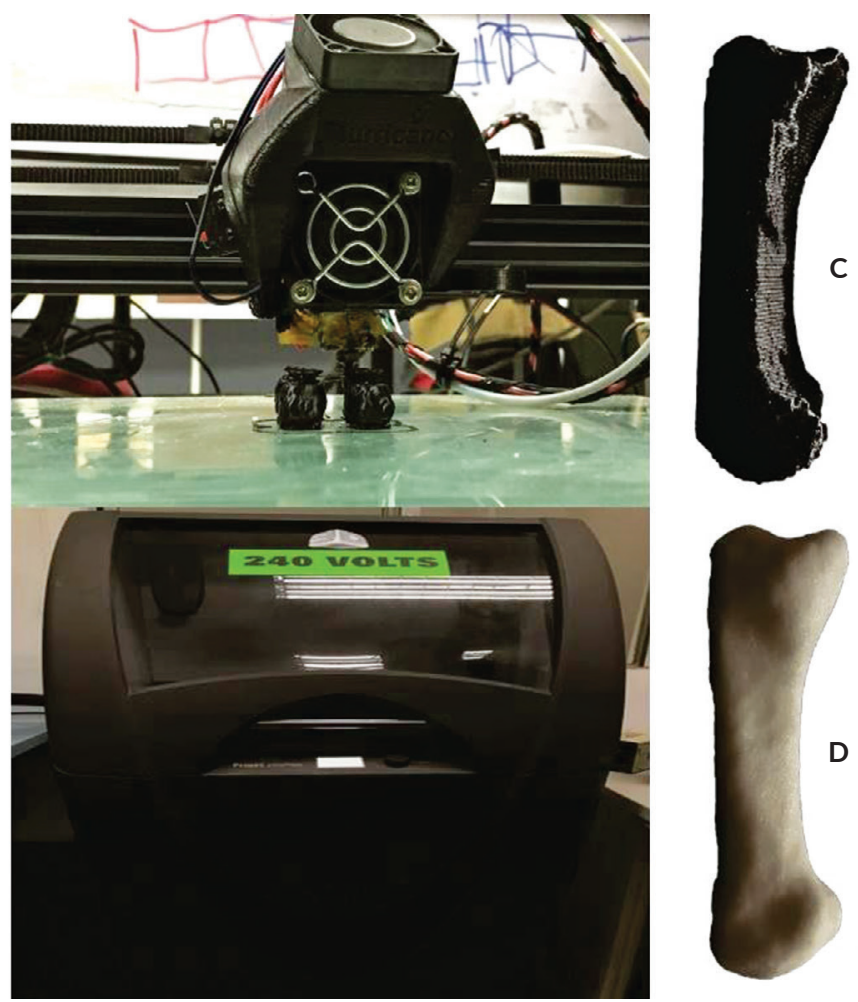

C

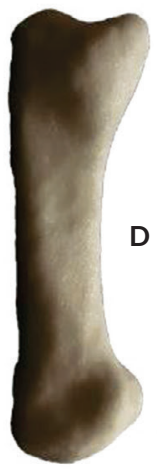

Figure 3. Four different 3D printers and corresponding models (A) Stereolithography, (B) Digital light processing, (C) Fused deposition method, and (D) Binder jetting.

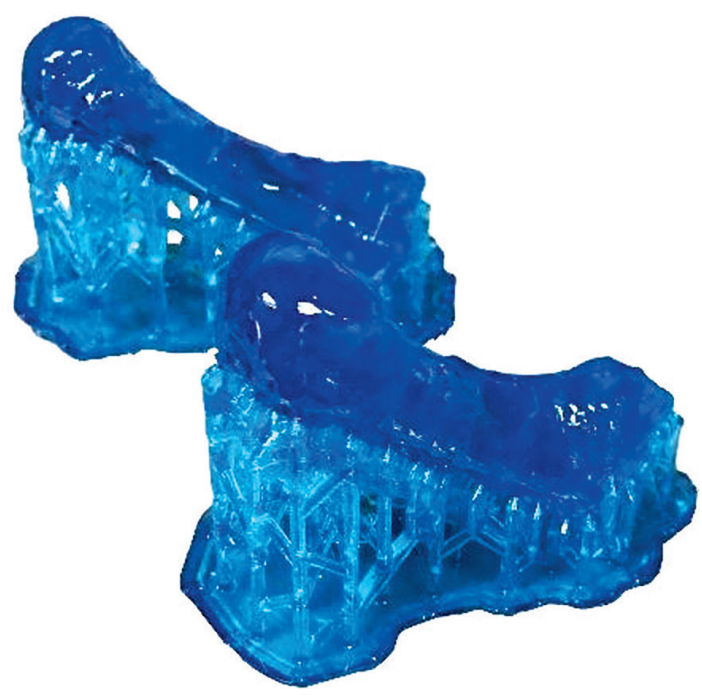

Figure 4. Support Structures on 3D-printed Model using Stereolithography.

\section{Data Processing and Analysis}

Consistent recording and data labelling were done. Mean measurements for the three replications for each parameter for each of the 3D-printed models and control bones were calculated using Microsoft Excel 2016.

Overall mean measurements for each parameter for all 3D-printed models and control bones were generated for the statistical analysis.
Non-inferiority testing was used to compare the overall mean measurements of each parameter between the 3D-printed models (experimental group) and the cadaveric bone (control). The differences in overall means and confidence intervals between cadaveric bones ( $\mu$ Bone) and 3D-printed models ( $\mu$ Model) determined whether the 3D-printed models were non-inferior to the cadaveric metacarpals. The non-inferiority limit, which is 0.5 , was the margin that was clinically acceptable to conclude that there is no statistically significant difference between the two groups. ${ }^{5}$

The null hypothesis (Ho) in this study states that $3 \mathrm{D}$-printed bones are inferior to cadaveric bones in terms of the difference in means. In contrast to this, the alternative hypothesis $(\mathrm{Ha})$ claims that $3 \mathrm{D}$-printed bones are noninferior to cadaveric bones in terms of the differences in means.

\section{$\mathrm{H}_{\mathrm{o}}$ : Difference in means $=\mu$ Bone $-\mu$ Model $>0.5$ \\ $\mathrm{H}_{\mathrm{a}}$ : Difference in means $=\mu$ Bone $-\mu$ Model $\leq 0.5$}

We also used multidimensional scaling to determine how similar the 3D-printed models (using different printing methods) and control bones are to one another. Unlike the non-inferiority testing, which was restricted to comparing measurements between two groups, multidimensional scaling compared all groups, and portrayed their similarities or differences in a graph. ${ }^{6}$ 


\section{Ethical Considerations}

This study was approved by the University of the Philippines Manila Research Ethics Board (UPM-REB). Written consent to use the cadavers was obtained from the Department of Anatomy, College of Medicine, University of the Philippines Manila. Only the right first metacarpals were used in order to preserve the dignity of the cadavers. In addition, the bones were labelled, stored, and were returned properly to each body after measurement of identified parameters.

\section{RESULTS AND DISCUSSION}

Mean measurements of 3D-printed models for each parameter for each printing method were slightly larger than their control bone measurements, except for the head height measurement of DLP which is $0.114 \mathrm{~mm}$ less than the control bone (Table 2). Standard deviation for all parameters ranged from 1.219 to 1.939 , except for the length which had a higher range of 3.766 to 4.264 . This was also observed in the standard error for all parameters, where only length had a higher standard error (1.044 to 1.183) compared to the others. However, the measurements of the 3D-printed models were not far from the control bone, with an accuracy of at least $90 \%$. The base width of DLP-printed models had the highest accuracy (99.62\%). In addition, DLP also yielded the most accurate measurements across all parameters while binder jet method yielded the least accurate measurements.

The mean differences $(-1.387$ to 0.114$)$ showed that the measurements of all the 3D-printed models are greater than those of the control bones (Figure 5). The confidence intervals of each comparison did not exceed the upper boundary of 0.5 . This indicates that the $3 \mathrm{D}$-printed models are not inferior to the control bones for all parameters.

Using multidimensional scaling and a Shepard diagram to show how similar the various $3 \mathrm{D}$-printed models are to the bone, the DLP-printed model (0.437) was the most accurate and has the closest similarity to the bone (0.899) (Figure 6). This was followed by FDM (-0.085), binder jetting $(-0.456)$ and lastly, SLA (-0.794). Despite the difference in the accuracy of each 3D printing method, all 3D-printed models from all four printing methods were non-inferior to their control bone.

The non-inferiority of 3D-printed models implies that the four printing methods are suitable for recreating and printing simple anatomical structures in three dimensions. However, this does not imply that they are exactly equivalent to the anatomical bones that were used. The disparity and discrepancies behind the different accuracies of these $3 \mathrm{D}$ printing methods could be attributed to a lot of factors, ranging from pre- and post-3D printing processing. Creating a model from 3D printing involves a lot of specific processes where possible sources of variability come from. An example would be software rendering, where CT scan
Table 2. Accuracy and Mean Measurements of Bones and 3D-Printed Models per Dimensional Parameter

\begin{tabular}{|c|c|c|c|c|c|}
\hline Parameter & Code & $\begin{array}{c}\text { Mean } \\
\text { (in } \mathrm{mm} \text { ) }\end{array}$ & $\begin{array}{l}\text { Standard } \\
\text { Deviation }\end{array}$ & $\begin{array}{l}\text { Standard } \\
\text { Error }\end{array}$ & $\begin{array}{c}\text { Accuracy* } \\
\text { (\%) }\end{array}$ \\
\hline \multicolumn{6}{|c|}{$\begin{array}{l}\text { Length } \\
\end{array}$} \\
\hline Control bone & LO & 44.85 & 3.77 & 1.04 & Reference \\
\hline Binder jet & L1 & 45.79 & 4.26 & 1.18 & 97.90 \\
\hline DLP & L2 & 45.67 & 4.08 & 1.13 & 98.17 \\
\hline FDM & L3 & 45.98 & 4.24 & 1.18 & 97.48 \\
\hline STL & L4 & 46.24 & 4.15 & 1.15 & 96.91 \\
\hline \multicolumn{6}{|c|}{ Midshaft diameter } \\
\hline Control bone & MSO & 10.55 & 1.40 & 0.39 & Reference \\
\hline Binder jet & MS1 & 11.29 & 1.23 & 0.34 & 92.91 \\
\hline DLP & MS2 & 11.06 & 1.22 & 0.34 & 95.13 \\
\hline FDM & MS3 & 11.25 & 1.33 & 0.37 & 93.27 \\
\hline STL & MS4 & 11.56 & 1.31 & 0.36 & 90.35 \\
\hline \multicolumn{6}{|c|}{ Base width } \\
\hline Control bone & BWO & 13.88 & 1.60 & 0.44 & Reference \\
\hline Binder jet & BW1 & 14.35 & 1.62 & 0.45 & 96.65 \\
\hline DLP & BW2 & 13.94 & 1.54 & 0.43 & 99.62 \\
\hline FDM & BW3 & 14.13 & 1.50 & 0.42 & 98.19 \\
\hline STL & BW4 & 14.43 & 1.56 & 0.43 & 96.09 \\
\hline \multicolumn{6}{|c|}{ Base height } \\
\hline Control bone & $\mathrm{BHO}$ & 14.86 & 1.41 & 0.39 & Reference \\
\hline Binder jet & $\mathrm{BH} 1$ & 15.49 & 1.56 & 0.43 & 95.79 \\
\hline DLP & $\mathrm{BH} 2$ & 14.98 & 1.48 & 0.41 & 99.21 \\
\hline FDM & $\mathrm{BH} 3$ & 15.30 & 1.54 & 0.43 & 97.08 \\
\hline STL & $\mathrm{BH} 4$ & 15.69 & 1.43 & 0.40 & 94.41 \\
\hline \multicolumn{6}{|c|}{ Head width } \\
\hline Control Bone & HWO & 14.94 & 1.69 & 0.47 & Reference \\
\hline Binder Jet & HW1 & 15.67 & 1.60 & 0.44 & 95.13 \\
\hline DLP & HW2 & 15.12 & 1.67 & 0.46 & 98.80 \\
\hline FDM & HW3 & 15.27 & 1.55 & 0.43 & 97.80 \\
\hline STL & HW4 & 15.72 & 1.60 & 0.45 & 94.81 \\
\hline \multicolumn{6}{|c|}{ Head height } \\
\hline Control bone & HHO & 13.00 & 1.85 & 0.51 & Reference \\
\hline Binder Jet & $\mathrm{HH} 1$ & 13.41 & 1.94 & 0.54 & 96.79 \\
\hline DLP & $\mathrm{HH} 2$ & 12.88 & 1.92 & 0.53 & 99.12 \\
\hline FDM & $\mathrm{HH} 3$ & 13.28 & 1.89 & 0.52 & 97.82 \\
\hline STL & $\mathrm{HH} 4$ & 13.53 & 1.88 & 0.52 & 95.93 \\
\hline
\end{tabular}

DLP, Digital light processing; FDM, Fused deposition modelling; STL, Stereolithography

* \%Accuracy $=$ Relative difference $\times 100$

and model rendering are intimate procedures. Parameters of the CT scan directly impacts the model that is rendered. In a previous study, it was noted that one of the sources of inaccuracy in biomedical fabrication is the scanning variables, specifically slice thickness. ${ }^{3}$ The slice thickness used during the study may not have been the optimal setting for $3 \mathrm{D}$-printing a right metacarpal bone. Aside from this, modelrendering involves hole-filling, mesh-editing, smoothing, and self-intersecting inspection, which are user-dependent, and hence, may be a source of variation. 
Comparison

L0 vs L1
L0 vs L2
L0 vs L3
L0 vs L4
MS0 vs MS1
MS0 vs MS2
MS0 vs MS3
MS0 vs MS4
BW0 vs BW1
BW0 vs BW2
BW0 vs BW3
BW0 vs BW4
BH0 vs BH1
BH0 vs BH2
BH0 vs BH3
BH0 vs BH4
HW0 vs HW1 1
HW0 vs HW2
HW0 vs HW3
HW0 vs HW4
HH0 vs HH1
HH0 vs HH2
HH0 vs HH3
HH0 vs HH4

Difference $(90 \% \mathrm{Cl})$

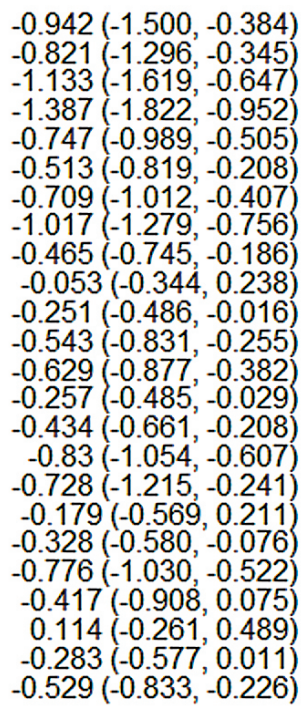

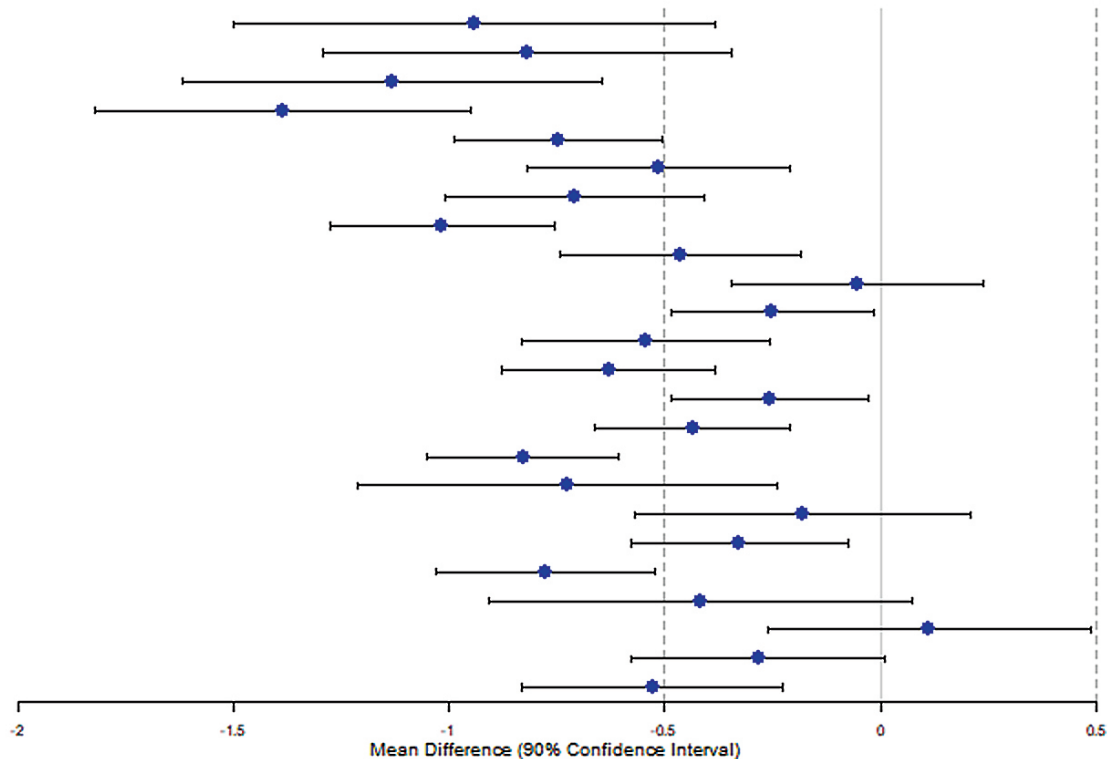

L, Length; MS, Midshaft diameter; BW, Base width; BH, Base height; HW, Head width; HH, Head height; 0, Bone; 1, Binder jet; 2, DLP; 3, FDM; 4, STL

Figure 5. Non-inferiority testing comparing bone and 3D-printed model measurements.

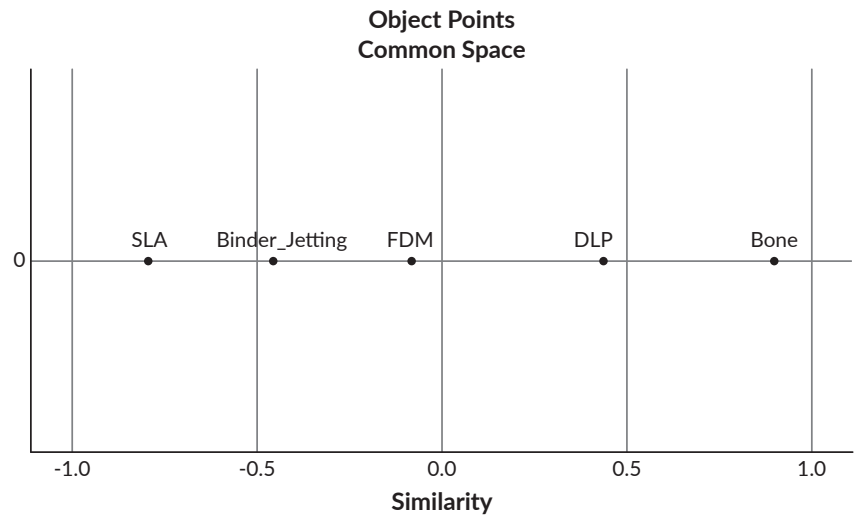

SLA, Stereolithography; FDM, fused deposition modeling; DLP, Digital light processing

Figure 6. Shepard diagram comparing the different 3D-printed models to control bone.

However, if it is assumed that no problems have been encountered in software-rendering since the CT scan files of all the bones used were uniform for all $3 \mathrm{D}$ printers, it could appear that discrepancies would be widely dependent on two factors: specific 3D printing method used and post-processing. ${ }^{4}$

$3 \mathrm{D}$ printing in the current study may have affected the results given that the $3 \mathrm{D}$ printers have different procedures that involves a lot of human intervention. Some of the models had to be cured - which involved handling while at a deformable state, especially for the hardening process of the binder jetting method. Removal of support structures for the SLA and DLP may also be considered as a source of variability. Aside from the human intervention, thermal deformation of the material during storage and warping of the models can be sources of variation. ${ }^{4,7}$

In terms of the printing method, DLP and SLA are similar in that they both use a photosensitive liquid bath. ${ }^{8,9}$ The main difference is the use of ultraviolet light with 60-70 degrees Celsius ambient temperature in SLA, while DLP uses a spatial light modulating element., ${ }^{8,9}$ These factors result in warping during the post-processing of 3D-printed models. ${ }^{7}$ In warping, there will be differences in the outcome of 3D-printed models as parameters such as length or width could shrink or expand; thus causing other parameters to adjust by shrinking and expanding as well. ${ }^{7}$ The post-processing of DLP only entailed an alcohol bath and running water, while SLA utilized alcohol bath and cured with ultraviolet light. This additional exposure may have led to thermal stress and thus significantly affected the measurements of SLA-printed models. ${ }^{4}$

Post-processing of FDM and binder jet may have also affected the dimensions of their 3D-printed models. The process of FDM utilizes a layering method wherein succeeding layers are squeezed on top of the previous layer. ${ }^{9}$ In addition, FDM uses a support which still has to be manually removed either by hand or a tool. ${ }^{9}, 10$ According to a previous study, the additional factor of a using a support structure makes the 3D-printed model highly vulnerable to inaccuracies. ${ }^{4}$

On the other hand, binder jetting does not employ support structures but still follows a layer-by-layer method of additive production similar to FDM..${ }^{11}$ During the actual post-processing, binder jet-printed models were cured with 
a hardener, which may have had adverse effects on the dimensions of the models. This may explain why this method yielded the least accurate measurements among all printing methods, Nonetheless, it was still found to be non-inferior compared to the bone.

\section{Study Limitations}

Selection bias may have occurred in choosing the cadavers and in the outcome assessors who volunteered to participate in our study due to logistical and time constraints. On the part of the researchers, selection bias was minimized through randomization of cadavers used in the study. In addition, inter-observer variability may have been present due to the fact that the set of volunteer outcome assessors for bone measurement were different from those set for measurement of the $3 \mathrm{D}$ printed models. However, this was reduced by thorough supervision of the volunteer outcome assessors by the investigators during the measurements. Detection and measurement bias were also minimized through standardization and calibration of measuring tools, standardization of measurement methods, and blinding of the outcome assessors.

\section{CONCLUSION}

3D-printed models of the right first metacarpal bones were accurate; they were non-inferior to the control bones in terms of six measurement parameters. DLP-printed models were found to be the most accurate for all parameters and the most similar compared to the bone. Minor differences can be due pre- to post-3D printing processing (e.g., removal of support structures), post-processing treatments, slice thickness, thermal stress, and warping or shrinking. 3D-printed models may help in assisting medical and other health professionals in their respective fields, specifically those requiring accurate templating using models.

\section{Recommendations}

For future studies on 3D printing, we recommend using more complex bones, such as the pelvis, cranium, and scapula. We also recommend the use of digital measurements utilizing $\mathrm{CT}$ scan software and DICOM files. There is also a need to compare parameters other than the external measurements, such as medullary cavity diameter, cortical thickness, and density. Since 3D printing is a relatively new field in surgical practice here in the Philippines, the researchers also recommend evaluation of the cost-effectiveness of the process in this setting.

\section{Acknowledgments}

The researchers would like to express their gratitude to: Dr. Mark Christian E. Manuel, Lorenz Toleos, and Bradly Lee Lomotan of Mapua University; the Department of Anatomy, College of Medicine, UP Manila; the faculty and staff of the Radiology Department of UP-PGH Faculty
Medical Arts Building; the IDC 211 and 211.1 panel and advisers; and all the volunteers who took part in this study.

\section{Statement of Authorship}

All authors participated in data collection and analysis, and approved the final version submitted.

\section{Author Disclosure}

All authors declared no conflicts of interest.

\section{Funding Source}

None.

\section{REFERENCES}

1. Lal H, Patralekh MK. 3D printing and its applications in orthopaedic trauma: A technological marvel. J Clin Orthop Trauma. 2018 Jul-Sep; 9(3):260-8.

2. Ventola CL. Medical applications for 3D printing: Current and projected uses. PT. 2014 Oct; 39(10):704-11.

3. Fitzwater KL, Marcellin-Little DJ, Harrysson OL, Osborne JA, Poindexter EC. Evaluation of the effect of computed tomography scan protocols and freeform fabrication methods on bone bio-model accuracy. Am J Vet Res. 2011 Sep; 72(9):1178-85.

4. Zou Y, Han Q, Weng X, Zou Y, Yang Y, Zhang K, et al. The precision and reliability evaluation of 3- dimensional printed damaged bone and prosthesis models by stereo lithography appearance. Medicine. 2018 Feb; 97(6):e9797.

5. Case DT, Rawlins CM, Mick CB. Measurement standards for human metacarpals. Am J Phys Anthropol. 2015 Jun; 157(2):322-9.

6. NCSS LLC. Multidimensional Scaling. NCSS 2019 Software Manual [Internet]. 2006 [cited 2019 Jun 2]. Chapter 435. Available from: https://ncss-wpengine.netdna-ssl.com/wp-content/themes/ncss/pdf/ Procedures/NCSS/Multidimensional_Scaling.pdf

7. Parab ND, Barnes JE, Zhao C, Cunningham RW, Fezzaa K, Rollett $\mathrm{AD}$, et al. Real-time observation of binder jetting printing process using high-speed X-ray imaging. Sci Rep. 2019 Feb; 9(1):2499.

8. High Resolution SLA and SLS 3D Printers for Professionals [Internet]. 2012. SLA vs. DLP: A 3D Printing Technology Comparison. Formlabs. 2017 [cited 2019 Jun 2]. [about 2 paginations]. Available from: https://formlabs.com/blog/3d-printing-technologycomparison-sla-dlp/

9. All3DP [Internet]. 2019. DLP vs SLA - 3D Printing Technologies Shootout. Pires, R. 2018 [cited 2019 Jun 2]. Available from: https:// all3dp.com/2/dlp-vs-sla-3d- printing-technologies-shootout/

10. 3D Hubs [Internet]. 2019. Introduction to FDM 3D printing. Varotsis, A. 2017 [cited 2019 Jun 2]. Available from: https://www.3dhubs. com/knowledge- base/introduction-fdm-3d-printing

11. Additive Manufacturing [Internet]. 2018. AM 101: Binder Jetting. Arnold, K. 2019 [cited 2019 Jun 2]. [about 8 paginations]. Available from: https://www.additivemanufacturing.media/blog/post/ am-101-binder-jetting

12. Cone JA, Martin TM, Marcellin-Little DJ, Harrysson OL, Griffith EH. Accuracy and repeatability of long-bone replicas of small animals fabricated by use of low-end and high-end commercial threedimensional printers. Am J Vet Res. 2017 Aug; 78(8):900-5.

13. Hwang HH, Zhu W, Victorine G, Lawrence N, Chen S. 3D-printing of functional biomedical microdevices via light- and extrusionbased approaches. Small Methods. 2018 Feb; 2(2):1700277.

14. Mardis NJ. Emerging technology and applications of $3 \mathrm{D}$ printing in the medical field. Mo Med. 2018 Jul-Aug; 115(4):368-73.

15. Garcia J, Yang Z, Mongrain R, Leask RL, Lachapelle K. 3D printing materials and their use in medical education: A review of current technology and trends for the future. BMJ Simul Technol Enhanc Learn. 2018 Jan; 4(1):27-40. 
Dimensional Accuracy of 3D-printed Metacarpal Models

\section{APPENDIX}

\section{Description of 3D printers}

Four 3D printers, each with its unique machine and material, were used in producing the anatomical models for the study.

\section{Stereolithography (SLA)}

A photoreactive liquid resin bath is selectively exposed to ultraviolet (UV) light to form thin solid layers that stack up to create a 3D product. A platform, onto which the product is printed, is moved vertically at a speed of $1-3 \mathrm{~cm} / \mathrm{hr}$., depending on the size of the cross-sectional area on the build plate, through the liquid substrate as the UV laser is being projected in order to allow an additive manner of printing. The polymerization approach of this process is highly localized, utilizing points and lines that are fed to the two motors or galvanometers of the machine as a set of coordinates ( $\mathrm{X}$ and $\mathrm{Y}$ axis). As a result, the printing process is slow. ${ }^{12}$

\section{Digital light processing (DLP)}

Similar to SLA technology, a photosensitive liquid resin is used. In contrast to SLA, however, it uses a spatial light modulating (SLM) element or a digital projection screen, which is easier to maintain and is cheaper than UV light. A single image of each layer of the model is projected by the element and is reproduced onto the liquid substrate at a rate of around 2.6 inches/hr. vertical print. Whereas the light hits the resin on a single spot in SLA, the whole layer is formed at once on DLP. ${ }^{13}$ Each layer is built by rectangular bricks called voxels since the process is dependent on pixels. This usage of pixels limits DLP method when producing small details of models with large build volume since their pixel size is limited. ${ }^{12}$

Both DLP and SLA use tough resin as building material. Tough resin has high strength and compliance which makes it the best choice for prototyping functional parts that undergo periods of strength and stress. SLA is ideal for printing many small intricate parts at once such as concept modeling, rapid prototyping and art models. In comparison, DLP is used in printing large parts without much detail such as parts for functional prototypes and mechanical assemblies. The post-processing methods for each technology are also different. In DLP, it is enough for the 3D model to be submerged in an alcohol bath then washed with running water. On the other hand, a 3D model printed with SLA has to be cured with UV light at 60-70 degrees Celsius ambient temperature after the alcohol bath to make it more mechanically stable. ${ }^{12}$

\section{Fused deposition modeling (FDM)}

This process utilizes a wide selection of filaments such as acrylonitrile butadiene styrene (ABS), poly-lactic acid (PLA), nylon polyamide (Nylon PA), and glycol modified polyethylene terephthalate (PET-G). The material used in the study was black ABS filament with $1.75 \mathrm{~mm}$ diameter, which was melted and extruded through the machine's nozzle onto a building platform. The extrusion head is attached to a 3-axis system that allows it to build each layer on top of the other in the X, $\mathrm{Y}$ and $\mathrm{Z}$ directions. The advantages of $\mathrm{ABS}$ are good strength and temperature resistance but its distinct disadvantage is susceptibility to warping. ${ }^{14}$

FDM is the most widely used 3D printing technology since it is the most cost- effective way of producing custom thermoplastic parts and prototypes, has a wide range of thermoplastic materials available, and has short lead times while being the most available technology. However, it is limited by its low dimensional accuracy and resolution, the need for postprocessing cleaning for a smooth finish, and by its layer adhesion mechanism which makes FDM parts anisotropic. ${ }^{14}$

\section{Binder jetting}

The binder jetting method utilizes several materials such as metals, sand, and ceramics. In the study, we used a proprietary powder substrate that was placed on a vertically movable platform and formed a product using a liquid binding agent. A ProJet CJP 260Plus was used specifically for the study that has 5-10 times faster print speeds and up to 7 times lower cost than all other 3D printing technologies. In this process, a thin layer of powder is first spread by a re-coater on the machine's build platform. The printhead or nozzle will then spray the binding agent onto the powder according to the object's geometry. This deposition and binding are done layer by layer until the whole part is complete. After printing, excess powder is cleaned from the model using compressed air before a hardener is applied to strengthen and further solidify it. The whole process is done in a controlled environment minimizing the probability of warping or curling. ${ }^{15}$ 